\title{
Comparative Prospective Study Reporting Intraoperative Parameters, Pedicle Screw Perforation, and Radiation Exposure in Navigation-Guided versus Non-navigated Fluoroscopy-Assisted Minimal Invasive Transforaminal Lumbar Interbody Fusion
}

\author{
Tarun Dusad, Vishal Kundnani, Shumayou Dutta, Ankit Patel, Gaurav Mehta, Mahendra Singh \\ Department of Orthopaedics, Bombay Hospital \& Medical Research Centre, Mumbai, India
}

\begin{abstract}
Study Design: Prospective cohort study.
Purpose: To compare intraoperative parameters, radiation exposure, and pedicle screw perforation rate in navigation-guided versus non-navigated fluoroscopy-assisted minimal invasive transforaminal lumbar interbody fusion (MIS TLIF).

Overview of Literature: The poor reliability of fluoroscopy-guided instrumentation and growing concerns about radiation exposure have led to the development of navigation-guided instrumentation techniques in MIS TLIF. The literature evaluating the efficacy of navigation-guided MIS TLIF is scant.

Methods: Eighty-seven patients underwent navigation- or fluoroscopy-guided MIS TLIF for symptomatic lumbar/lumbosacral spondyIolisthesis. Demographics, intraoperative parameters (surgical time, blood loss), and radiation exposure (sec/mGy/Gy.cm² noted from $\mathrm{C}$-arm for comparison only) were recorded. Computed tomography was performed in patients in the navigation and non-navigation groups at postoperative 12 months and reviewed by an independent observer to assess the accuracy of screw placement, perforation incidence, location, grade (Mirza), and critical versus non-critical neurological implications.

Results: Twenty-seven patients (male/female, 11/16; L4-L5/L5-S1, 9/18) were operated with navigation-guided MIS TLIF, whereas 60 (male/female, 25/35; L4-L5/L5-S1, 26/34) with conventional fluoroscopy-guided MIS TILF. The use of navigation resulted in reduced fluoroscopy usage (dose area product, $0.47 \mathrm{~Gy} . \mathrm{cm}^{2}$ versus $2.93 \mathrm{~Gy} . \mathrm{cm}^{2}$ ), radiation exposure (1.68 mGy versus $10.97 \mathrm{mGy}$ ), and fluoroscopy time (46.5 seconds versus 119.08 seconds), with $p$-values of $<0.001$. Furthermore, $96.29 \%$ (104/108) of pedicle screws in the navigation group were accurately placed (grade 0 ) (4 breaches, all grade I) compared with $91.67 \%(220 / 240)$ in the non-navigation group (20 breaches, 16 grade I+4 grade II; $p=0.114)$. None of the breaches resulted in a corresponding neurological deficit or required revision.

Conclusions: Navigation guidance in MIS TLIF reduced radiation exposure, but the perforation status was not statistically different than that for the fluoroscopy-based technique. Thus, navigation in nondeformity cases is useful for significantly reducing the radiation exposure, but its ability to reduce pedicle screw perforation in nondeformity cases remains to be proven.
\end{abstract}

Keywords: Minimal invasive surgical procedures; Spondylolisthesis; Fusion; Computer-assisted; Radiation exposure

Received May 14, 2017; Revised Jul 28, 2017; Accepted Aug 24, 2017

Corresponding author: Tarun Dusad

Spine Clinic, Shop no. 3, 1st floor, Onkar Towers, Shivdham Sankul, Gen AK Vaidhya Marg, Across Oberoi Mall, Malad (E), Mumbai, India Tel: +91-7710935049, Fax: +91-22-22080871, E-mail: tarundusad@gmail.com 


\section{Introduction}

Transforaminal lumbar interbody fusion (TLIF) is the favored tool of spine surgeons for symptomatic lumbar and lumbosacral spondylolisthesis. Morbidity, blood loss, postoperative infection, prolonged hospitalization stay, and prolonged rehabilitation can be avoided by performing the surgery in a minimally invasive manner, i.e., using minimal invasive TILF (MIS TLIF). However, there can be a higher chance of screw misplacement and neurological implications [1-5] when using this method due to the absence of visible anatomic landmarks and reduced tactile feedback [1].

The conventional fluoroscopic technique for pedicle screw placement results in both the patient and surgeon being exposed to a considerable amount of radiation. The effects of radiation can be acute or deterministic and late or stochastic [6], such as skin erythema, skin necrosis, cataract formation, malignancies, and heritable disorders. The amount of radiation exposure for the patient is accepted as being within the safe range for a singular event. However, for spine surgeons, the occupational lifetime exposure can be significant with uncertain consequences. Studies have examined spinal instrumentation in cadavers, phantoms for documenting radiation burdens on patients [7-9], and the impact of radiation exposure on surgeons using dosimetry [10]. Analyzing surgeons' radiation exposure using dosimetry has certain limitations: it is dependent on the exposed area and does not consider cumulative radiation exposure, which is a more specific variable in the analysis of radiation exposure, both direct and scattered.

The poor reliability of fluoroscopy-guided instrumentation and growing concerns about radiation exposure have led to the development of navigation-guided instrumentation techniques. The advantages of fluoroscopy can be expanded and the disadvantages eliminated by combining fluoroscopic techniques with computer-assisted navigation technology. However, studies comparing navigated and non-navigated MIS TLIF are scant.

The objective of this study was thus to compare radiation exposure, instrumentation accuracy, and intraoperative parameters in navigation-guided versus non-navigated MIS TLIF.

\section{Materials and Methods}

\section{Patients}

Following ethical committee approval, a prospectively maintained surgical registry of patients who underwent single level lumbar or lumbosacral MIS TLIF was reviewed (Table 1). A total of 87 patients were included after meeting the strict inclusion and exclusion criteria (Table 2). The patient cohort was then stratified into two groups: patients who underwent navigation-guided MIS TLIF (study group: 27 patients; L4-L5/L5-S1, 9/18) and those who underwent conventional fluoroscopy-guided nonnavigated MIS TLIF (control group: 60 patients; L4-L5/ L5-S1, 26/34). All the surgeries were performed between November 2010 and December 2014, and patients were followed up at 3, 6, and 12 months.

Inclusion criteria were as follows: (1) age 20-80 years, (2) single level lumbar or lumbosacral symptomatic spondylolisthesis grade $\leq 3$, and (3) failed conservative trial of 3 months. Exclusion criteria were as follows: (1) age $<20$ years or $>80$ years, (2) multilevel spinal instability, (3) no radiculopathy, (4) previous spinal surgery, (5) spinal trauma or infection, and (6) patient did not try a conservative trial for 3 months.

All surgeries were performed by a single fellowship trained spine surgeon. The surgeon started performing fluoroscopy-based MIS TLIF after gaining experience in open TLIF and other simple microscopic procedures and started performing navigation-based MIS after getting well-acquainted with navigation setup and equipment. This should minimize the surgeon's experience-based bias in the current study. Patients were consecutively selected to undergo MIS TLIF. Initial cases were performed only with fluoroscopy guidance. Later, patients were allotted to either group according to the patient's choice and affordability and availability of a navigation setup in the hospital.

\section{Clinical evaluations}

A prospective analysis of the patients' demographics and intraoperative parameters was performed. This analysis included factors such as age, sex, body mass index, diagnosis, operated level, operating time, intraoperative blood loss, notable events (e.g., dural leak), pain scores, neurological status, and complications. 


\section{Surgical technique: navigation-guided minimal inva- sive transforaminal lumbar interbody fusion}

Under general anesthesia, the patient was positioned prone on a radiolucent table. The computer-assisted navigation system consisted of a calibration target attached to a C-arm, navigation workstation (StealthStation S7; Medtronic Inc., Minneapolis, MN, USA), an optical camera to detect the patient tracker, and specific instruments (dynamic reference array, pointer, pedicle awl, and pedicle probe) equipped with passive reflectors to be detected by the optical camera. Decompression was performed using a 24-mm dilator. A metallic/PEEK (polyetheretherketone) cage was inserted from the side that showed worse symptoms.

A dynamic reference array was attached to the patient's iliac bone at posterior superior iliac spine. Serial true anteroposterior (AP) and lateral images were obtained and transferred to the computer. The optical camera measures the relative position of the $\mathrm{C}$-arm and patient at the time of image acquisition by detecting the dynamic reference array. After calibration, the computer generates a graphic overlay to show the relative position of the instruments with respect to fluoroscopic images. The incision was made with the help of the navigation pointer. The pedicles were cannulated on both sides using a navigated awl, and guide wires were passed in all four pilot holes. The pedicles were tapped with the navigation instrument, and pedicle screws of appropriate length and diameter were placed on both sides. Appropriately sized rods were placed percutaneously and fixed with pedicle screws. Final AP and lateral fluoroscopic images were recorded before the wound was irrigated and closed. Intraoperative data, which included the surgery time (including navigation system setup time), blood loss, number of pedicle screws, level of fixation, intraoperative complications, cumulative radiation exposure, dose area product (DAP), and radiation time, were then noted.

In all patients, a common C-arm (BV Endura; Philips, Eindhoven, The Netherlands) designed to automatically control radiation was used. Dose summaries generated by the $\mathrm{C}$-arm were used to measure the radiation exposure and include cumulative radiation exposure (mGy), DAP $\left(\mathrm{Gy} . \mathrm{cm}^{2}\right)$, and radiation time (seconds).

Cumulative radiation exposure (air kerma) is defined as the kinetic energy per unit mass of air delivered to a defined point in space. It is measured in mGy. It does not account for the area exposed or the patient's location with respect to the $\mathrm{C}$-arm, and may thus overestimate or underestimate the actual peak skin dose [11].

DAP is defined as the product of the patient's skin dose and area of skin irradiated within the collimated radiation field. It is automatically measured in Gy. $\mathrm{cm}^{2}$ by the DAP meter fixed in the $\mathrm{C}$-arm. It accounts for the exposed area and acquisitions. Its value is independent on the spatial plane and thus accounts for patient location. It correlates best with the effective dose [11].

Radiation time is the total time for which the X-ray beam is activated. It is measured in seconds. It does not account for the exposed area or acquisition. It is least correlated with patient dose and clinical outcomes [11].

\section{Radiological evaluations}

All patients underwent computed tomography (CT) at postoperative 12 months; an early CT was considered mandatory in cases of worsening postoperative radiculopathy or neurological deficits. Fusion and screw positioning were evaluated simultaneously with a single CT-associated radiation exposure. All the scans were reviewed twice for the following checkpoints by an independent fellowship trained spine surgeon and the chief spine surgeon: (1) accuracy of pedicle screw placement and percentage of accurately placed screws; (2) perforation incidence (number of perforation/total number of screws placed); (3) location of perforation (pedicle border perforated [medial/ lateral/superior/inferior]); (4) grade of perforation as per Mirza et al. [12] (grade I, $<2 \mathrm{~mm}$ breach; grade II, $>2 \mathrm{~mm}$ breach; grade III, entire screw outside the pedicle); and (5) critical versus non-critical breach (critical breach, the screw comes in contact with the dura or nerve root and causes injury; non-critical breach, a perforation with no critical contact).

All observations concerning perforation were confirmed by the radiologist and chief spine surgeon. CTs were performed in automatic exposure control (AEC) mode using $2 \mathrm{~mm}$ cuts to reduce the risk of radiation hazards.

\section{Statistical analysis}

Statistical analyses were performed using the SPSS ver. 10.2 (SPSS Inc., Chicago, IL, USA) software. The Student $t$-test was used to compare continuous variables. The data 
are presented as the mean \pm standard deviation. For all analyses, a $p$-value of $<0.05$ was considered significant.

\section{Results}

The current study included 87 patients (51 females and 36 males) with symptomatic (mechanical and radicular) lumbar or lumbosacral spondylolisthesis who underwent MIS TLIF; 27 were operated using navigation-guided MIS TLIF (study group; L4-L5/L5-S1, 9/18), whereas 60 using conventional fluoroscopy without navigation (control group; L4-L5/L5-S1, 26/34). Five patients in the non-navigation group were initially allotted to the navigation group, but due to some technical problems, the fluoroscopy-based technique was used instead. The mean age was $57.94 \pm 8.292$ years in the navigation group and $57.15 \pm 7.780$ years in the non-navigation group, with a male/female ratio of 11/16 and 25/35, respectively (Table 1 ).

Navigation-guided MIS TLIF required longer surgical time than that in conventional MIS TLIF (mean time, $166.11 \pm 16.588$ minutes and $154 \pm 10.076$ minutes, respec- tively; $p<0.05)$, and the difference was statistically significant (Table 3). The difference in time may be attributed to the navigation system set up time, acquisition of images, and learning curve for surgeons and staff.

The mean intraoperative blood loss was $128.89 \pm 21.389$ $\mathrm{mL}$ and $124.77 \pm 27.15 \mathrm{~mL}$ in the navigation and nonnavigation groups, respectively, with a $p$-value of 0.30 , i.e., difference was not statistically significant (Table 3 ). No intraoperative complications, such as dural leak or neurological injury, were observed.

The radiation indices were noted from the C-arm after completion of the procedure. The mean DAP was $0.470 \pm 0.122 \mathrm{~Gy} . \mathrm{cm}^{2}$ in the navigation group and $2.928 \pm 0.870 \mathrm{~Gy} . \mathrm{cm}^{2}$ in the non-navigation group, with a $p$-value of $<0.00001$. The mean cumulative radiation exposure was $1.68 \pm 0.419 \mathrm{mGy}$ and $10.97 \pm 2.38 \mathrm{mGy}$ in the navigation and non-navigation groups, respectively, and the difference was statistically significant $(p<0.00001)$. The mean radiation time was $46.50 \pm 11.89$ seconds in the navigation group and $119.08 \pm 29.784$ seconds in the nonnavigation group, with $p<0.00001$ (Table 3 ).

Table 1. Demographics of patients

\begin{tabular}{lcc} 
Variable & Navigation group & Non-navigation group \\
\hline Mean age (yr) & $57.94 \pm 8.292$ & $57.15 \pm 7.780$ \\
\hline Sex (male/female) & $11 / 16$ & $25 / 35$ \\
\hline Mean body mass index $\left(\mathrm{kg} / \mathrm{m}^{2}\right)$ & $28.92 \pm 2.76$ & $27.18 \pm 3.77$ \\
\hline Symptoms & Mechanical and claudication, radiculopathy & Mechanical and claudication, radiculopathy \\
\hline Level L4-L5/L5-S1 & $9 / 18$ & $26 / 34$ \\
\hline Total no. of screws & 108 & 240 \\
\hline Diagnosis & Deg./lytic spondylolisthesis & Deg./lytic spondylolisthesis
\end{tabular}

Values are presented as mean \pm standard deviation, unless otherwise stated.

Deg., degenerative.

Table 2. Inclusion and exclusion criteria

Inclusion criteria

Age 20-80 yr

Single level lumbar or lumbosacral symptomatic spondylolisthesis $\leq$ grade 3

Conservative trial tried for at least for 3 months

\section{Exclusion criteria}

Age $<20$ or $>80$ yr

Multilevel spinal instability or spondylolisthesis grade 4 or 5

No radiculopathy

Previous spine surgery

Spinal trauma or infection

Conservative trial not tried for at least 3 months 
In all the patients, immediate relief of leg pain was reported with some persistence of back pain. No recurrence of leg pain or worsening of back pain was reported by any patient at the 12-month follow-up.

All the patients underwent CT at the end of one year for assessment of accurate pedicle screw placement and fu-

Table 3. Comparison of intra-operative parameters

\begin{tabular}{lccc} 
Outcome feature & Navigation used (N=27) & Navigation not used (N=60) & $p$-value \\
\hline Surgical time (min) & $166.11 \pm 16.58$ & $154 \pm 10.077$ & $<0.05$ \\
\hline Blood loss (mL) & $128.89 \pm 21.389$ & $124.77 \pm 27.15$ & 0.30 \\
\hline Dose area product (Gy.cm ${ }^{2}$ ) & $0.470 \pm 0.122$ & $2.93 \pm 0.87$ & $<0.05$ \\
Cumulative radiation dose (mGy) & $1.68 \pm 0.419$ & $10.97 \pm 2.38$ & $<0.05$ \\
\hline Radiation time (sec) & $46.5 \pm 11.89$ & $119.075 \pm 29.78$ & $<0.05$ \\
\hline
\end{tabular}

Values are presented as mean \pm standard deviation.
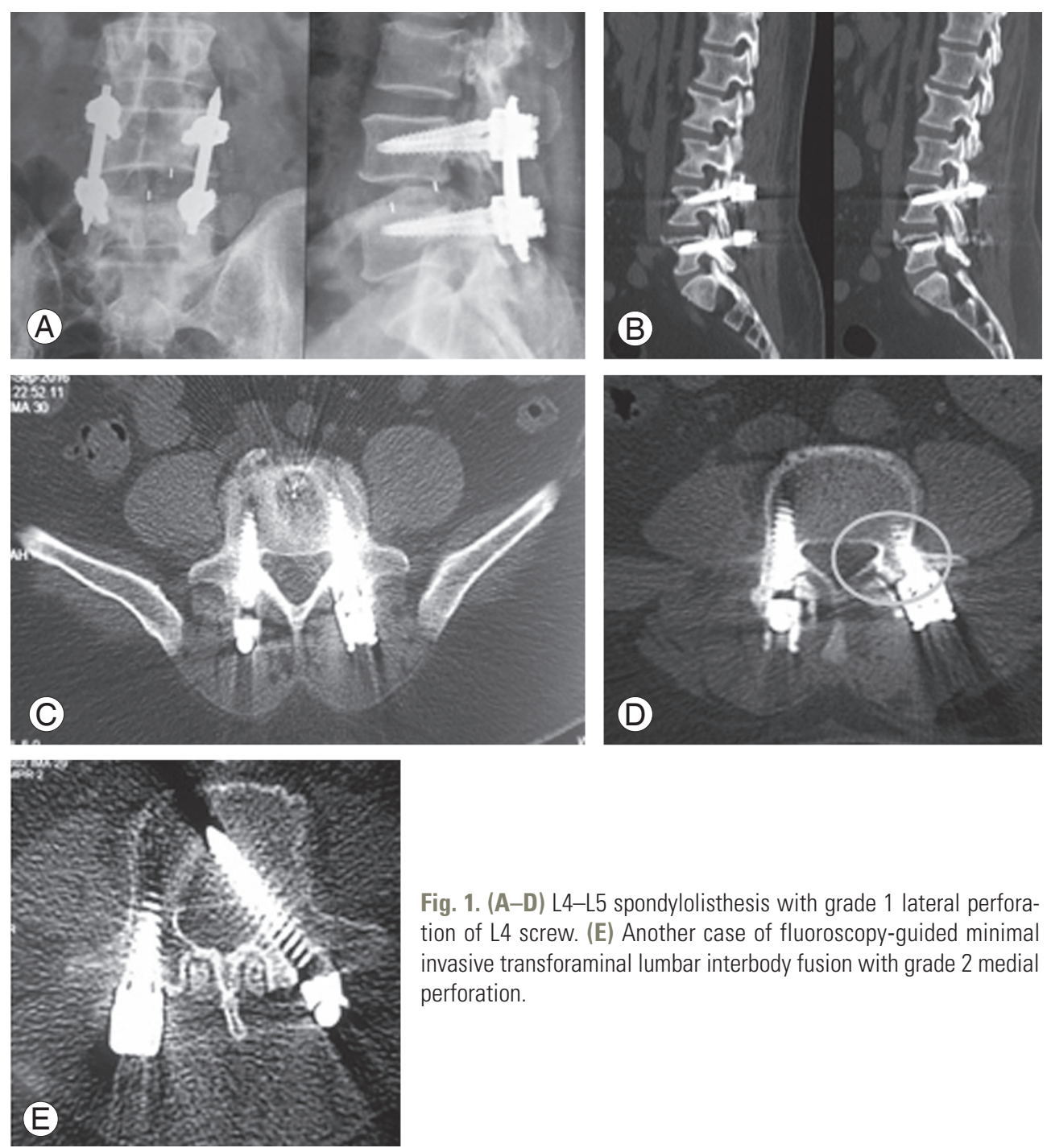

Fig. 1. (A-D) $L 4-L 5$ spondylolisthesis with grade 1 lateral perforation of $L 4$ screw. (E) Another case of fluoroscopy-guided minimal invasive transforaminal lumbar interbody fusion with grade 2 medial perforation. 
Table 4. Pedicle screw perforation status of navigation-guided versus non-navigation minimal invasive transforaminal lumbar interbody fusion

\begin{tabular}{lccc} 
Parameter & Navigation group & Non-navigation group & Significance \\
Pedicle perforation & & & $p>0.05$ \\
\hline Grade 1 & $4 / 108$ & $16 / 240$ & \\
\hline Grade 2 & 0 & $4 / 240$ & \\
Grade 3 & 0 & 0 & \\
\hline
\end{tabular}

sion. As fusion was not a part of the current study, we have not provided a detailed explanation; we simply noted that there was no statistical difference between the two groups. It was found that $96.29 \%(104 / 108)$ of the pedicle screws in the navigation group were accurately placed (grade 0 ) compared with $91.67 \%(220 / 240)$ in the non-navigation group. There were four and 16 grade 1 breaches for the navigation and non-navigation groups, respectively (Fig. 1). Four grade 2 and no grade 3 pedicle breaches were observed in the non-navigation group, whereas no grade 2 or 3 screws were noted in the navigation group ( $p=0.114$ ) (Table 4). The number of pedicle breaches was more in the non-navigation than in the navigation group, but the difference was not statistically significant. Furthermore, $50 \%$ of breaches in the navigation group were lateral and $50 \%$ were medial, whereas these values were $60 \%$ and $40 \%$ in the non-navigation group, respectively. None of the breaches were critical, and none resulted in a corresponding neurological deficit or required revision.

\section{Discussion}

MIS TLIF has been known to improve the fusion rate and restore alignment and disc height while avoiding complications associated with open technique, such as surgical soft tissue trauma, excess blood loss, postoperative pain, and prolonged rehabilitation time [13]. However, this technique cannot compensate for the loss of visual anatomical landmarks and tactile feedback while positioning pedicle screws. MIS TLIF is technically more demanding with a steep learning curve, and there are growing concerns about the cumulative radiation exposure associated with this technique. We combined navigation guidance with MIS TLIF to treat symptomatic lumbar or lumbosacral spondylolisthesis and compared its effectiveness with conventional fluoroscopy-based MIS TLIF.

A navigation system allows for the visualization of the current position of a surgical instrument without the need to capture multiple fluoroscopic images and reposition the $\mathrm{C}$-arm during the procedure. Navigation permits overlay of the instrument graphic over preacquired fluoroscopic images in multiple planes simultaneously. In the current study, we observed that the cumulative radiation dose was significantly higher in non-navigated MIS TLIF. Accurate dose measurement can be very difficult because it depends on various factors, including radiation time, patient-source distance, patient-receptor distance, magnification, AEC, surgical procedure, position of surgical members, and radiation protection methods. Complete emitted radiation is thus a better measure for comparing fluoroscopy usage between the two groups. DAP is most closely related with the effective dose of radiation, and it was 6.23 times higher in the non-navigation group. Navigation along with radiation protection principles can significantly reduce long-term radiation exposure and aid in reducing overall radiation exposure for patients and surgeons.

The navigation technique may be helpful for increasing the accuracy and convenience of pedicle screw placement in MIS TLIF. In the current study, $96.29 \%(104 / 108)$ of pedicle screws in the navigation group were accurately placed (grade 0) compared with $91.67 \%(220 / 240)$ in the non-navigation group. The number of pedicle breaches was higher in the non-navigation than in the navigation group, but the comparison was not statistically significant $(p=0.114)$. None of the breaches were critical and did not cause any neurological deficit or required revision. Navigation allows the visualization of multiple fluoroscopic images representing the spinal anatomy at a single time, and the surgeon can choose the optimal entry point, trajectory, length, and diameter of screws with precision; however, the difference in the number of pedicle breaches was not statistically significant between the two groups in this study. A larger cohort should be studied to prove the significance of navigation in lowering screw perforation where spinal anatomy is relatively preserved, i.e., low- 
grade spondylolisthesis cases.

The results of the current study are consistent with the findings of the prior literature. Ravi et al. [1] evaluated the clinical accuracy of two-dimensional (2D) computerassisted fluoroscopy for percutaneous placement of lumbosacral pedicle screws. In this study, the fluoroscopy time for screw placement was $<20 \mathrm{sec} / \mathrm{case}$. The authors reported no breach or an acceptable breach $(<2 \mathrm{~mm})$ in $96.3 \%$ of cases in navigation-guided MIS TLIF. Villard et al. [10] compared occupational radiation exposure of surgeons and patients during posterior lumbar spine instrumentation in 10 navigated cases versus 11 cases using the free-hand technique. The authors observed that three-dimensional (3D) fluoroscopy-based spinal navigation used for lumbar fusion significantly reduced the surgeon's radiation exposure by up to 9.96 times (1884.8 cGy.cm ${ }^{2}$ [non-navigated] versus $887 \mathrm{cGy} . \mathrm{cm}^{2}$ [navigated]). Rampersaud et al. [14] assessed the radiation exposure of spine surgeon during fluoroscopy-assisted thoracolumbar pedicle screw placement. They reported that conventional fluoroscopy-based pedicle screw placement was associated with 10-12 times greater radiation exposure for the surgeon than that in non-spinal orthopedic procedures. Smith et al. [15] compared the radiation dose for the surgeon during pedicle screw insertion with 3D fluoroscopybased navigation and conventional fluoroscopic guidance in a cadaveric study with no measurable radiation exposure in most cases of navigation assistance. Kim et al. [8], in a similar sort of cadaveric study, determined that fluoroscopic time was significantly reduced due to navigation.

Yang et al. [16] compared the accuracy and utility of computer-assisted fluoroscopic navigation for percutaneous placement of lumbar pedicle screws with conventional fluoroscopic placement. They observed 3\% and 7.2\% breaches with and without navigation, respectively. Tian et al. [13] evaluated the clinical and radiological outcomes of computer-assisted minimally invasive spine surgery TLIF and open TLIF for the treatment of one-level degenerative lumbar diseases. They used 3D CT-based navigation and reported that the accuracy of pedicle screw placement was 93.33\% with navigation-guided MIS TLIF. Ringel et al. [17] assessed the feasibility and safety of percutaneous posterior pedicle screw fixation for instabilities of the thoracic and lumbar spine and reported $13 \%$ of $>2$-mm pedicle breach rate with conventional fluoroscopic technique.

The mean surgical time for the navigation group was $166.11 \pm 16.588$ minutes compared with $154 \pm 10.076$ min- utes for the non-navigation group. The navigation system set up time, acquisition of images, and registration and learning curves of the surgeon and staff for navigation may contribute to the total surgical time in the navigation group.

In the current study, there was $128.89 \pm 21.389 \mathrm{~mL}$ and $124.77 \pm 27.15 \mathrm{~mL}$ blood loss in the navigation and nonnavigation groups, respectively, without any statistically significant difference $(p=0.30)$. None of the patients required blood transfusion. Similarly, Yang et al. [16] reported that the estimated blood loss in $71.6 \%$ patients was $<200 \mathrm{~mL}$ without any significant difference between the navigation versus non-navigation groups.

The current study had some limitations. First, the number of patients included in this study was small. As such, this study may be underpowered and may represent a limited assessment of pedicle screw breach in patients. A larger cohort is required to establish the significance of navigation techniques, especially regarding screw perforation in cases such as MIS TLIF. Second, this was a single institute, single surgeon-based study, which may have limited the generalizability of the results that can vary with the experience of the surgeon.

Third, there are some limitations of 2D fluoroscopybased navigation. It can neither update the guide wire position while tapping and placing screws nor help in cage placement where fluoroscopic images are captured. Proper fluoroscopic techniques, such as centring the area of interest within the field of view and tangential imaging (true AP and lateral), are foremost requirements for navigation-guided MIS TLIF. The navigation system set up time, acquisition of images, and their registration may add to the total surgical time. Additionally, 2D navigation does not provide multiplanar detailed imaging. Fixing of the reference arc is invasive, which constrains the surgeon's movement and puts its own stability at risk. It also cannot compensate for causes of poor image quality, such as obesity. In difficult cases, it may not be as accurate as $3 \mathrm{D}$ navigation.

\section{Conclusions}

The use of navigation in spinal surgery has improved the safety of spinal instrumentation. Further improvement in navigation technology will expand its applications in spine surgery. This technology, however, is not a substitute for thorough knowledge of spinal anatomy and 
traditional methods of instrumentation. MIS TLIF is a commonly performed procedure in cases of spondylolisthesis. Navigation in MIS TLIF has the added advantage of significantly reducing the radiation exposure during surgery compared with conventional fluoroscopy-guided MIS TLIF, and further growth in this field is anticipated to track guide wires and aid in interbody cage placement. Its significance in reducing pedicle screw perforation in nondeformity and low-grade spondylolisthesis cases is yet to be proved. Hence, its utility in lowering radiation exposure must be balanced against the time and cost of surgery for low-grade spondylolisthesis.

\section{Conflict of Interest}

No potential conflict of interest relevant to this article was reported.

\section{References}

1. Ravi B, Zahrai A, Rampersaud R. Clinical accuracy of computer-assisted two-dimensional fluoroscopy for the percutaneous placement of lumbosacral pedicle screws. Spine (Phila Pa 1976) 2011;36:84-91.

2. Kosmopoulos V, Schizas C. Pedicle screw placement accuracy: a meta-analysis. Spine (Phila Pa 1976) 2007;32:E111-20.

3. Tian NF, Xu HZ. Image-guided pedicle screw insertion accuracy: a meta-analysis. Int Orthop 2009;33:895903.

4. Esses SI, Sachs BL, Dreyzin V. Complications associated with the technique of pedicle screw fixation: a selected survey of ABS members. Spine (Phila Pa 1976) 1993;18:2231-8.

5. Lonstein JE, Denis F, Perra JH, Pinto MR, Smith MD, Winter RB. Complications associated with pedicle screws. J Bone Joint Surg Am 1999;81:1519-28.

6. International Commission on Radiological Protection. 1990 Recommendations of the International Commission on Radiological Protection. Oxford: Pergamon Press; 1991.

7. Gebhard FT, Kraus MD, Schneider E, Liener UC, Kinzl L, Arand M. Does computer-assisted spine surgery reduce intraoperative radiation doses? Spine (Phila Pa 1976) 2006;31:2024-7.

8. Kim CW, Lee YP, Taylor W, Oygar A, Kim WK. Use of navigation-assisted fluoroscopy to decrease radia- tion exposure during minimally invasive spine surgery. Spine J 2008;8:584-90.

9. Slomczykowski M, Roberto M, Schneeberger P, Ozdoba C, Vock P. Radiation dose for pedicle screw insertion: fluoroscopic method versus computer-assisted surgery. Spine (Phila Pa 1976) 1999;24:975-82.

10. Villard J, Ryang YM, Demetriades AK, et al. Radiation exposure to the surgeon and the patient during posterior lumbar spinal instrumentation: a prospective randomized comparison of navigated versus non-navigated freehand techniques. Spine (Phila Pa 1976) 2014;39:1004-9.

11. Hernanz-Schulman M, Emmons M, Price R. Fluoroscopy clinical practice: controlling dose and study quality. In: Frush DP, Huda H, editors. Categorical course syllabus in diagnostic radiology physics: from invisible to visible: the science and practice of X-ray imaging and radiation dose optimization. Oak Brook (IL): Radiological Society of North America; 2006. p.133-9.

12. Mirza SK, Wiggins GC, Kuntz C 4th, et al. Accuracy of thoracic vertebral body screw placement using standard fluoroscopy, fluoroscopic image guidance, and computed tomographic image guidance: a cadaver study. Spine (Phila Pa 1976) 2003;28:402-13.

13. Tian W, Xu YF, Liu B, et al. Computer-assisted minimally invasive transforaminal lumbar interbody fusion may be better than open surgery for treating degenerative lumbar disease. Clin Spine Surg 2017;30:237-42.

14. Rampersaud YR, Foley KT, Shen AC, Williams S, Solomito M. Radiation exposure to the spine surgeon during fluoroscopically assisted pedicle screw insertion. Spine (Phila Pa 1976) 2000;25:2637-45.

15. Smith HE, Welsch MD, Sasso RC, Vaccaro AR. Comparison of radiation exposure in lumbar pedicle screw placement with fluoroscopy vs computer-assisted image guidance with intraoperative three-dimensional imaging. J Spinal Cord Med 2008;31:532-7.

16. Yang BP, Wahl MM, Idler CS. Percutaneous lumbar pedicle screw placement aided by computer-assisted fluoroscopy-based navigation: perioperative results of a prospective, comparative, multicenter study. Spine (Phila Pa 1976) 2012;37:2055-60.

17. Ringel F, Stoffel M, Stuer C, Meyer B. Minimally invasive transmuscular pedicle screw fixation of the thoracic and lumbar spine. Neurosurgery 2006;59(4 Suppl 2):ONS361-6. 